\title{
Superstars and the Long Tail: The impact of technology on market structure in media industries
}

\author{
Helen Weeds \\ University of Essex*
}

25 January 2012

\begin{abstract}
Technological change has transformed creative media industries. Digitization lowers the costs of recording, storage, reproduction and distribution, while computer-based editing facilitates quality enhancement and special effects. Digital technology has altered the distribution of sales in ways that remain poorly understood: while some commentators have highlighted the growth of the "long tail", others find digitization has raised the importance of "superstars". This paper develops a theoretical model of differentiated goods with endogenous quality to investigate the impact of digitization on the distribution of firms. It finds that supply-side factors can generate superstars and long tail outcomes, and that coexistence of both phenomena can be explained by either a fall in fixed costs for basic products or a decline in market size.
\end{abstract}

*Mailing address: Department of Economics, University of Essex, Wivenhoe Park, Colchester CO4 3SQ, UK. Email: hfweeds@essex.ac.uk. 
Keywords: Digital media; creative industries; superstars; long tail.

JEL codes: L11, L15, L82.

\section{Introduction}

Digitization has transformed creative media industries, with a range of impacts on the cost structure of production and supply. In the production of recorded music and video content, digitization has lowered the costs of recording, storage and reproduction. Computer-based editing has made higher quality production possible at lower cost and facilitated new special effects superior to those previously available. Distribution on physical media has shifted to cheaper, more compact, higher quality formats - from vinyl and tape to CD for music; from VHS to DVD and Blu-ray for video-while electronic distribution over cable and the internet has greatly reduced distribution costs by eliminating the transportation of physical media altogether. Digitization of television signals has permitted many more channels to be shown for a given capacity of radio spectrum or cable infrastructure, and allowed images to be broadcast in higher definition. Online stores have entered retail markets: with lower fixed costs than traditional outlets these are able stock a far larger number of products, making a potentially vast range of varieties available to consumers.

Technological developments have also affected the demand side of creative media industries. New formats and modes of distribution, such as e-books, movie downloads, and mobile television, have expanded demand in some areas by offering consumers new ways of enjoying media products. Internet and electronic distribution have enabled retailers to serve harder-to-reach customers who struggle to access high street stores, and to supply national and global markets rather than just the local area. However, recent developments have not always been beneficial for producers: in the music industry, where the ease of copying and storing digital music has facilitated widespread 
file-sharing and piracy, commercial sales have fallen dramatically.

Digital technology has altered the distribution of sales in creative media industries in ways that remain poorly understood. Some commentators have highlighted a shift towards niche products and the growth of the "long tail". Anderson $(2004,2006)$ reports that online music distributors stock many more albums than a typical high street outlet, with even relatively unpopular tracks achieving a handful of downloads each month, and also notes the decline in sales levels for the top music albums in the early 2000s. Other authors report a more mixed picture, finding in addition to the long tail the presence of "superstar" effects as conceived by Rosen (1981), which found that the shift from live to recorded music increased returns to top artists. In a study of (legal) online music downloads, Page and Garland (2009) find a "hitheavy, skinny-tail" distribution, with more than three-quarters of the total inventory of tracks remaining unsold. Similarly, Elberse and Oberholzer-Gee (2007) find that in the US home video market (DVD and VHS), sellers offer more products but many of these achieve few sales, while amongst best-sellers there is greater concentration on a small number of titles.

This paper investigates theoretically the impact of digitization on the distribution of firms in creative media industries. The goal is to characterize the distribution of products supplied under free entry conditions where products are differentiated both horizontally and vertically. To do this I develop a model that captures key features of creative media industries, and use this to study the effects of technological changes that lower distribution costs, fixed costs, and the cost of enhancing product quality. Specifically, I adapt the "pyramid" spatial model developed by Von Ungern-Sternberg (1991) and simplified by Brito (2003), in which competition is symmetric between products, in contrast to the "near neighbor" form of the more familiar Salop (1979) framework. The equilibrium number and mix of heterogeneous products is then determined by a free entry condition.

Market structure is characterized in the following way. Superstar out- 
comes occur when there is greater investment in the quality of top-selling or "premium" products (e.g. higher expenditure on blockbuster movies), and/or these products achieve higher individual sales, either in absolute terms or as a share of the market. In other words, superstar phenomena are associated with "bigger hits". The long tail refers to an increase in the number of niche or "basic" products that are sold; for this to be more than an empty tail, basic products must also account for an increased share of total sales.

The main insight of the paper is that both superstar and long tail outcomes can be explained by supply-side factors. Moreover, in some situations the two phenomena can coexist, consistent with the empirical findings described above. Results show that a reduction in fixed costs for basic products generates both a long tail, increasing the number and share of niche products sold, and superstar effects, raising the quality of premium products and the sales of each one. A decline in market size also generates both types of effects. By contrast a reduction in the cost of enhancing quality is one-sided, raising investment in premium products - a superstar effect - but not a long tail.

This research contributes to a small but growing literature on long tail effects. Brynjolfsson, $\mathrm{Hu}$ and Simester (2011) focus on consumer search, examining how a reduction in search costs affects concentration in product sales. In a theoretical model of consumer search behavior lower search costs result in a less concentrated sales distribution, a result which they observe empirically in sales data from a multi-channel retailing company. Fleder and Hosanagar (2009) use analytical modeling and simulation to examine the effect of recommender systems on sales diversity. They find that while these systems may increase individual-level diversity, they tend to push consumers towards the same products, decreasing aggregate diversity. Tucker and Zhang (2011) assess the effect of popularity information on consumer choice, under the hypothesis that the same level of popularity implies higher quality for narrow-appeal products (niches) than for broad-appeal products that would 
be expected to attract a large number of customers. Using data from a website listing wedding service vendors, the authors find that niche vendors receive more clicks than equally popular broad-appeal vendors, softening the concentration bias of popularity-based recommendations.

In focusing on search costs and recommender systems, this literature emphasizes demand-side technological drivers of long tail effects. By contrast, this paper emphasizes supply-side factors, in particular the role of technology in altering exogenous and endogenous fixed costs.

The paper is structured as follows. Section 2 develops a model of differentiated products with endogenous quality and free entry, initially with identical cost functions. This is extended to heterogeneous products in section 3 : here firms choose between the production of basic and premium goods, which have different production technologies. Using this framework, the impact of developments linked to digitization on industry structure and outcomes are examined. Section 4 concludes. An appendix contains longer proofs.

\section{A model of horizontal differentiation with endogenous quality}

\subsection{Modeling approach}

In modeling the impact of digitization in creative media industries, I wish to capture the following features and developments:

1. Horizontal differentiation: media content is a highly diverse product class; consumers are heterogeneous in their individual preferences and most desire some variety of products. The impact of digitization on the number of product types supplied is central to the analysis.

2. Fixed costs: content production costs are almost entirely fixed, with a large "first copy" cost. Retailing also incurs some fixed costs, e.g. 
buildings and display space. Digitization lowers fixed production costs with cheaper video hardware, storage and editing, while internet retailing greatly reduces the fixed costs of stocking a range of products compared with traditional stores.

3. Quality and endogenous fixed costs: while being fixed in relation to the number of consumers, production costs typically increase with higher quality, thus fixed costs are at least partially endogenous. Digitization lowers the cost of quality enhancement, due to e.g. digital processing, high definition, computer-based editing and movie special effects.

4. Distribution costs: retail distribution incurs some per-unit cost, e.g. pressing and delivering a CD or DVD, though this is small compared with the fixed cost of content production. Digitization lowers distribution costs, especially with the replacement of physical media delivery by electronic downloads.

5. Market size: digitization can expand markets, e.g. through geographic integration, or shrink them, e.g. due to piracy.

To handle product heterogeneity in a framework that allows analytical solution of a free entry equilibrium, I adapt a generalization of the Hotelling (1929) model that allows for symmetric competition between an arbitrary number of firms. The starting-point is the pyramid model developed by Von Ungern-Sternberg (1991), in which producers are located at the corners of a multidimensional pyramid and consumers uniformly distributed along the edges. The model assumes a fixed number of potential locations (i.e. the dimensionality of the pyramid is given), and the number of brands actually produced is a subset of this. Competition is symmetric, but demand curves are kinked because of the missing brands. Brito (2003) simplifies the pyramid model by assuming that the dimensionality of the pyramid matches the number of firms with no unused slots. The Brito version has the feature that the distribution of consumers is not invariant to the entry of firms. 
Section 2.2 presents the basic model. Its parameters capture the following developments brought about by digitization: lower per-consumer distribution costs, a lower cost of quality enhancement, and changes in market size. The model of section 3 extends this framework by allowing firms to choose between products of different qualities involving different production technologies. The later model is used to examine the strategic choices of firms and, with free entry, the mix and market shares of heterogeneous products in industry equilibrium.

\subsection{The model}

Each of $N \geq 2$ single-product firms is connected to every other by a Hotelling line, the length of which equals the mass of consumers between the pair. ${ }^{1}$ The total length of the $\frac{1}{2} N(N-1)$ lines equals the total mass of consumers $M>0$; it would be natural to normalize $M=1$ but the parameterization allows for the possibility of variation around this. Demand is uniformly distributed, thus the length of the line between each pair of firms is $m=$ $\frac{2 M}{N(N-1)}$. Consumers incur a linear per-unit transport cost $t>0$, and purchase from the firm that offers them the highest utility net of transport costs. For a given pair of firms $i, j$ offering utilities $u_{i}, u_{j}$ respectively, the indifferent consumer is located at $x_{i j}$ such that $u_{i}-t x_{i j}=u_{j}-t\left(\frac{2 M}{N(N-1)}-x_{i j}\right) ; i$ 's

\footnotetext{
${ }^{1}$ In other words, the density of consumers is constant. If the lines were instead taken to be fixed in length, entry would decrease the density of consumers and so would not reduce average transport costs (unlike in Salop). In this case a single firm would be socially optimal as entry merely duplicates fixed costs.
} 
demand on this sub-market is therefore given $b^{2}$

$$
x_{i j}=\frac{1}{2 t}\left(u_{i}-u_{j}+\frac{2 M}{N} \frac{t}{N-1}\right) .
$$

Summing across the $(N-1)$ sub-markets, total demand facing firm $i$ is given by $x_{i}=\sum_{j \neq i} x_{i j}$, which yields ${ }^{3}$

$$
x_{i}=\frac{M}{N}+\frac{(N-1)}{2 t}\left(u_{i}-\bar{u}_{-i}\right),
$$

where $\bar{u}_{-i}=\frac{1}{N-1} \sum_{j \neq i} u_{j}$. Note that market shares are equal if firms offer identical utilities, and the expression reduces to the standard Hotelling specification when $N=2$.

Utility $u_{i}$ from consuming product $i$ depends on product quality $q_{i}=$ $v_{0}+v_{i}$ and price $p_{i}$ as follows

$$
u_{i}=v_{0}+v_{i}-p_{i}
$$

The quality of a basic product $v_{0}$ is assumed to be sufficiently high that all consumers gain positive utility from purchasing, thus the market is covered. A firm can choose its (variable) quality $v_{i}$ by incurring a fixed cost $\frac{1}{2} \gamma v_{i}^{2}$. There is a marginal distribution cost $c$ of supplying each consumer.

Timing in the game is as follows. Firms first choose whether or not

\footnotetext{
${ }^{2}$ In principle a firm could also compete for consumers on lines to which it is not directly connected by offering a product that is more attractive than that of the intermediate firm, offsetting its locational advantage (just as in Salop a firm could win consumers located beyond the neighboring firm). The rival would then make no sales, thus in equilibrium would not enter. It is therefore assumed that this situation does not arise, and each sub-market is divided between the two firms located at either end.

${ }^{3}$ This formulation would be identical to Brito (2003) but for that paper's assumption of a quadratic transport cost, which results in a different demand expression. Brito's model does not give a closed-form solution for the equilibrium number of firms under free entry, so is not suitable for use in this paper.
} 
to enter the market; active firms and consumers locate as described above. ${ }^{4}$ Firms then compete for consumers, simultaneously setting quality $v$ and price $p$.

Firm $i$ 's profit is given by

$$
\pi_{i}=\left(\frac{M}{N}+\frac{(N-1)}{2 t}\left(u_{i}-\bar{u}_{-i}\right)\right)\left(p_{i}-c\right)-\frac{1}{2} \gamma v_{i}^{2}
$$

Its best responses in price and quality are

$$
\begin{aligned}
p_{i} & =\frac{t M}{N(N-1)}+\frac{1}{2}\left(v_{i}+\bar{p}_{-i}-\bar{v}_{-i}+c\right) ; \\
v_{i} & =\frac{1}{2 t \gamma}(N-1)\left(p_{i}-c\right)
\end{aligned}
$$

where $\bar{p}_{-i}$ and $\bar{v}_{-i}$ are defined analogously to $\bar{u}_{-i}$ above. With $N$ firms, equilibrium price and quality are

$$
p_{N}=\frac{2 t M}{N(N-1)}+c \quad \text { and } v_{N}=\frac{M}{\gamma N},
$$

giving per-firm profit of

$$
\pi_{i}=\frac{M^{2}}{N^{2}}\left(\frac{2 t}{(N-1)}-\frac{1}{2 \gamma}\right)
$$

With free entry, $\pi_{i}(N)=0$ and the equilibrium number of firms is

$$
N^{*}=4 t \gamma+1
$$

\footnotetext{
${ }^{4}$ The model structure implies that consumer locations are endogenous to the number of firms that enter (as in Brito, 2003). Such an assumption may be justified by the finding among marketers that consumers have difficulty forming preferences over unknown products (or sets of characteristics), and instead form preferences over the available set of products.
} 
and equilibrium price and quality are

$$
p^{*}=\frac{M}{2 \gamma(4 t \gamma+1)}+c \quad \text { and } v^{*}=\frac{M}{\gamma(4 t \gamma+1)} \text {. }
$$

It can be seen from these results that the distribution cost $c$ passes through in full to consumer prices, and has no effect on either the number of firms or quality investment.

\subsection{Social optimum}

For comparison the socially optimal quality per firm and number of firms are derived. With $N$ firms each providing a product of quality $v_{0}+v$, total consumer utility from consumption is $\left(v_{0}+v\right) M$, transport costs are $\frac{t M^{2}}{2 N(N-1)}, 5$ and production costs $N\left(\frac{1}{2} \gamma v^{2}\right)$, giving welfare of

$$
W=\left(v_{0}+v\right) M-\frac{t M^{2}}{2 N(N-1)}-N\left(\frac{1}{2} \gamma v^{2}\right)
$$

Given $N$, the optimal quality choice is $\frac{M}{\gamma N}$, the same as in the market equilibrium. The welfare-maximizing number of firms is ${ }^{6}$

$$
N^{W}=1+t \gamma+\sqrt{t \gamma(1+t \gamma)}
$$

The market generates more (fewer) firms than is socially optimal for $t \gamma>(<$ ) $1 / 8$. Note that competitive equilibrium $\left(N^{*} \geq 2\right)$ requires $t \gamma \geq 1 / 4$, which entails excess entry (this result is common in locational models of product differentiation, as shown by Bhaskar and To, 2004). As $\gamma \rightarrow \infty$ (in the limit, quality is fixed) the ratio $N^{*} / N^{W}$ rises towards 2 , the same as that found in

\footnotetext{
${ }^{5}$ With $N$ firms, each is a distance $\frac{2 M}{N(N-1)}$ from its rivals, thus a consumer is on average $\frac{M}{2 N(N-1)}$ from her nearest product. The average transport cost is then $\frac{t M}{2 N(N-1)}$. With mass of consumers $M$, total transport costs are therefore $\frac{t M^{2}}{2 N(N-1)}$.

${ }^{6}$ Taking the positive root, as required by the second order condition for a maximum.
} 
the Salop model. Thus with endogenous quality the excess entry property is partially mitigated, though not eliminated.

\subsection{Impact of digitization}

In this model three parameters capture the effects of digitization: a lower distribution cost, $c$; a lower cost of quality enhancement, $\gamma$; and changes in market size, $M$. The following proposition presents the impacts of these changes on industry outcomes (proofs are straightforward and are omitted).

Proposition 1 In the endogenous quality model, the effects of changes brought about by digitization are as follows.

(i) A lower distribution cost reduces prices, but has no effect on quality or the equilibrium number of firms $\left(\partial p^{*} / \partial c>0, \partial v^{*} / \partial c=\partial N^{*} / \partial c=0\right)$.

(ii) A lower quality cost raises both quality and price, and reduces the equilibrium number of firms $\left(\partial v^{*} / \partial \gamma<0, \partial p^{*} / \partial \gamma<0, \partial N^{*} / \partial \gamma>0\right)$.

(iii) Market expansion raises both quality and price, but has no effect on the equilibrium number of firms $\left(\partial v^{*} / \partial M>0, \partial p^{*} / \partial M>0, \partial N^{*} / \partial M=0\right)$.

These results suggest that the role of digitization in lowering distribution costs has no effect on industry structure or quality investment, but feeds through to lower consumer prices. This trend has indeed been observed throughout the late 20th and early 21 st centuries, as successive new formats make creative content available at lower prices.

By reducing the cost of quality enhancement, however, digitization has more wide-ranging effects, raising equilibrium quality and price while reducing the number of firms. This is an endogenous fixed cost effect: as quality becomes cheaper to provide, firms invest more in raising quality and their fixed costs increase. With larger endogenous fixed costs, this reduces the equilibrium number of firms. In addition, prices increase to recoup the higher fixed costs. 
Market expansion raises the marginal return to quality, with similar effects to a lower quality cost: firms invest more, raising equilibrium quality and price. As in Sutton (1991), the increase in endogenous fixed costs inhibits entry - which would otherwise be expected in response to market growth, if fixed costs were purely exogenous - in this case leaving the equilibrium number of firms unchanged.

Both a lower cost of quality enhancement and market expansion generate a superstar effect, in that firms raise product quality. By reducing the equilibrium number of firms, a lower quality cost also allows each to capture a larger share of the market, another superstar outcome.

\section{Competition with heterogeneous products}

The central question of this paper is how digitization affects the production of different types of products. Firms may invest in better production infrastructure to increase the quality of their output, and if firms make different choices a dispersion of product qualities will exist. Producers adopting alternative production strategies may be differently affected by technological changes that affect the cost of quality enhancement and other factors. By allowing for product heterogeneity the impact of digitization on different producers and its implications for product mix can be examined.

Suppose now that there are two types of product, "basic" and "premium", that involve different production technologies. Basic products incur a relatively low, exogenous fixed cost, while premium production requires both a higher fixed cost and further investment in raising product quality. An example might be a movie producer's choice between low budget video and expensive studio production, where the latter allows scope for greater quality enhancement. Firms are identical ex ante but may choose different production technologies, resulting in ex post heterogeneity. 


\subsection{The model}

Upon entering the market a firm chooses between production of a basic $(B)$ or premium $(R)$ product. To produce a basic product a firm pays a fixed cost $F>0$, supplying a product of minimum quality $v_{0},{ }^{7}$ and cannot raise quality further. ${ }^{8}$ A premium product has endogenous quality, where a product of quality $q^{i}=v_{0}+v_{i}$ incurs a total (exogenous + endogenous) fixed cost of $K+\frac{1}{2} \gamma\left(v_{i}\right)^{2}$ where $K \in(F, 2 F)$. It seems a reasonable assumption that higher quality production requires somewhat larger exogenous fixed costs, and if $K=F$ no basic products would be produced in equilibrium. For simplicity, the marginal distribution cost $c$ is normalized to zero; as demonstrated in section 2.4 a per-unit cost simply adds to prices and affects no other variables.

The game takes place as follows. First, firms make entry decisions, choosing simultaneously whether to enter the market and which type of product to produce. Then in the second stage firms compete by choosing their prices ( $p^{B}$ and $p^{R}$, for basic and premium products respectively) and, for premium products, their quality $v$.

Firm $i$, of type $g \in\{B, R\}$, anticipates that a proportion $\lambda$ of its rivals will be of type $R$ and $(1-\lambda)$ of type $B$, with the total number of active firms being $N \geq 2$. Following the approach set out in section 2.2 above, it faces demand given by

$$
x_{i}^{g}=\frac{M}{N}+\frac{(N-1)}{2 t}\left(u_{i}^{g}-\lambda \bar{u}_{-i}^{R}-(1-\lambda) \bar{u}_{-i}^{B}\right),
$$

where $u_{i}^{B}=v_{0}-p_{i}^{B}$ and $u_{i}^{R}=v_{0}+v_{i}-p_{i}^{R}$. Profit functions for basic and

\footnotetext{
${ }^{7}$ As in section 2.2 , basic quality $v_{0}$ is assumed sufficiently high to ensure full consumer participation.

${ }^{8} \mathrm{~A}$ related model with two product types involving different quality costs, in which firms discover their product type after entry, finds that the higher-cost type makes no quality investment at all (for details see the earlier working paper available from the author). Hence the assumption that the quality of basic products cannot be raised is not unduly restrictive.
} 
premium production are respectively

$$
\begin{aligned}
\pi_{i}^{B} & =X_{i}^{B} p_{i}^{B}-F \\
\pi_{i}^{R} & =X_{i}^{R} p_{i}^{R}-K-\frac{1}{2} \gamma v_{i}^{2} .
\end{aligned}
$$

The profit function for each product is concave in its price and, for a premium product, in quality, thus second order conditions for a maximum are satisfied. Best responses in price and quality are

$$
\begin{aligned}
p_{i}^{B} & =\frac{t M}{N(N-1)}+\frac{1}{2}\left((1-\lambda) \bar{p}_{-i}^{B}+\lambda\left(\bar{p}_{-i}^{R}-\bar{v}_{-i}\right)\right) \\
p_{i}^{R} & =\frac{t M}{N(N-1)}+\frac{1}{2}\left((1-\lambda) \bar{p}_{-i}^{B}+\lambda\left(\bar{p}_{-i}^{R}-\bar{v}_{-i}\right)+v_{i}\right) \\
v_{i} & =\frac{p_{i}^{R}}{2 t \gamma}(N-1)
\end{aligned}
$$

Given $N$ and $\lambda$, second stage equilibrium prices and quality are

$$
\begin{aligned}
p_{e}^{B} & =\frac{1}{(N-1)} 2 t(4 t \gamma-(N-1)) H \\
p_{e}^{R} & =\frac{1}{(N-1)} 8 \gamma t^{2} H>p_{e}^{B} \\
v_{e} & =4 t H
\end{aligned}
$$

where $H=\frac{M}{N(4 t \gamma-(N-1)(1-\lambda))}$. Per-firm profits for the two products are then

$$
\begin{aligned}
\pi^{B} & =\frac{1}{(N-1)} 2 t(4 t \gamma-(N-1))^{2} H^{2}-F \\
\pi^{R} & =\frac{1}{(N-1)} 8 \gamma t^{2}(4 t \gamma-(N-1)) H^{2}-K .
\end{aligned}
$$

At the entry stage, zero profit conditions $\left(\pi^{B}=0\right.$ and $\left.\pi^{R}=0\right)$ determine 
$N$ and $\lambda$, yielding the following equilibrium outcomes

$$
\begin{aligned}
N^{*} & =4 t \gamma\left(\frac{K-F}{K}\right)+1 ; \\
\lambda^{*} & =\frac{F}{(K-F)}\left(\frac{M K G}{4 t \gamma(K-F)+K}-1\right) \\
v^{*} & =2(K-F) G ; \\
p^{B *} & =F G ; \\
p^{R *} & =K G>p^{B *},
\end{aligned}
$$

where $G=\sqrt{\frac{K}{2 \gamma F(K-F)}}>0$. These solutions imply that $H=\frac{(K-F) G}{2 t}>0$.

It can be seen that as $F \rightarrow 0, \lambda^{*} \rightarrow 0$ and premium products are crowded out. As $K \rightarrow 0, \lambda^{*} \rightarrow 1$ and basic products are crowded out. To ensure a solution such that $\lambda \in[0,1]$ the following parameter restriction is required

$$
\frac{4 \gamma t(K-F)+K}{M G} \in[F, K] .
$$

From the above results, demands facing a single firm producing each product type are given by

$$
\begin{aligned}
x^{B} & =\frac{1}{G} ; \\
x^{R} & =\frac{K}{G F}>x^{B} .
\end{aligned}
$$

Expressions can readily be derived for the number of firms producing each product type, $N^{B}=\left(1-\lambda^{*}\right) N^{*}$ and $N^{R}=\lambda^{*} N^{*}$ for basic and premium products respectively, total demands $X^{g}=N^{g} x^{g}$, individual market shares $s^{g}=x^{g} / M$, and total market shares $S^{g}=X^{g} / M$, where $g \in\{B, R\}$.

Deriving welfare results for the model with heterogeneous products is challenging. Transport costs are complicated by the fact that Hotelling lines are split asymmetrically when the competing products are of different types, and welfare is affected by the proportions of basic and premium products as 
well as the number of firms. Closed form solutions for the optimal number and mix of products cannot be obtained, and it is highly unlikely that the equilibrium and socially optimal outcomes will coincide.

\subsection{Impact of digitization}

With heterogeneous firms the following parameters capture the effects of digitization: a lower fixed cost for basic products, $F$; a lower fixed cost for premium products, $K$; a lower cost of quality enhancement for premium products, $\gamma$; and changes to market size, $M$. The following proposition presents the impacts of these changes on industry outcomes; the results are summarized in Table 1.

Proposition 2 In the model with heterogeneous products, the effects of changes brought about by digitization are as follows.

(1) A lower basic fixed cost $F$ raises the equilibrium number of firms, reduces the proportion of premium products, raises the quality of premium products, and reduces the prices of both basic and premium products. Demand for an individual product of either type increases, while the total market share of basic products goes up.

(2) A lower premium fixed cost $K$ reduces the equilibrium number of firms, increases the proportion of premium products, reduces the quality of premium products, increases the price of basic products, and may either increase or decrease the price of premium products. Demand for an individual product of either type falls, while the total market share of basic products goes down.

(3) A lower cost of quality enhancement for premium products decreases the equilibrium number of firms, but increases the proportion of premium products, the quality of premium products, and the prices of both basic and premium products. Demand for an individual product of either type falls, as 
Table 1: Comparative statics in the model with heterogeneous products

\begin{tabular}{c|cccccccc}
\hline & $N^{*}$ & $\lambda^{*}$ & $v^{*}$ & $p^{B^{*}}$ & $p^{R^{*}}$ & $x^{B}, x^{R}$ & $s^{B}, s^{R}$ & $S^{B}$ \\
\hline$F$ & - & + & - & + & + & - & - & - \\
$K$ & + & - & + & - & - then + & + & + & + \\
$\gamma$ & + & - & - & - & - & + & + & + \\
$M$ & none & + & none & none & none & none & - & - \\
\hline
\end{tabular}

does the total market share of basic products.

(4) Market expansion has no effect on the equilibrium number of firms but increases the proportion of premium products. It has no effect on prices or quality, leaving individual product demands unchanged, but reduces individual shares of the now-larger market. The total market share of basic products goes down.

Proof. The proposition follows from the comparative statics results (derivations are given in the appendix).

(1) $\partial N^{*} / \partial F<0, \partial \lambda^{*} / \partial F>0, \partial v^{*} / \partial F<0, \partial p^{B *} / \partial F>0, \partial p^{R *} / \partial F>$ $0, \partial x^{B} / \partial F<0, \partial x^{R} / \partial F<0, \partial S^{B} / \partial F<0$.

(2) $\partial N^{*} / \partial K>0, \partial \lambda^{*} / \partial K<0, \partial v^{*} / \partial K>0, \partial p^{B *} / \partial K<0, \partial p^{R *} / \partial K<(>)$ 0 for $K<(>) 3 F / 2, \partial x^{B} / \partial K>0, \partial x^{R} / \partial K>0, \partial S^{B} / \partial K>0$.

(3) $\partial N^{*} / \partial \gamma>0, \partial \lambda^{*} / \partial \gamma<0, \partial v^{*} / \partial \gamma<0, \partial p^{B *} / \partial \gamma<0, \partial p^{R *} / \partial \gamma<0, \partial x^{B} / \partial \gamma>$ $0, \partial x^{R} / \partial \gamma>0, \partial S^{B} / \partial \gamma>0$.

(4) $\partial \lambda^{*} / \partial M>0, \partial N^{*} / \partial M=\partial v^{*} / \partial M=\partial p^{B *} / \partial M=\partial p^{R *} / \partial M=\partial x^{B} / \partial M=$ $\partial x^{R} / \partial M=0, \partial s^{B} / \partial M<0, \partial s^{R} / \partial M<0, \partial S^{B} / \partial M<0$.

\subsection{Discussion}

This model allows the implications of the following developments related to digitization to be examined: lower exogenous fixed costs for basic and 
premium products respectively, cheaper provision of quality, and changes in market size. The effects of a change in one parameter are often complex, with interactions between the variables: e.g. a change in exogenous fixed costs may alter quality investment (with no change in the cost of quality enhancement), affecting endogenous fixed costs and other industry outcomes.

\subsubsection{Fixed costs}

Recall that in Salop (1979) a reduction in exogenous fixed costs raises the equilibrium number of firms, increasing the range of varieties on offer. In this model, however, the incidence of fixed cost reductions for basic and premium products makes a crucial difference to this result. If digitization reduces the basic fixed cost $F$ (e.g. by lowering the cost of video recording) then the entry of basic products is encouraged. This increases both the equilibrium number of firms - raising variety, as in Salop - and the proportion of basic products $\left(1-\lambda^{*}\right)$. These are long tail effects. Moreover, in this case the long tail of basic products is not an empty one, with basic products accounting for a larger share of the total market (higher $S^{B}$ ).

By contrast, if it is the premium fixed cost $K$ that is reduced (e.g. lower costs of high-end studio production) then the opposite result is found: the equilibrium number of firms falls, reducing variety. This somewhat surprising result can be explained as follows. All else being equal, a reduction in fixed costs for premium products stimulates their entry, increasing the proportion of premium products. But this leaves less room for basic products, inhibiting their entry. Since, from (14), the demand for an individual premium product exceeds that of a basic product, entry of an additional premium product squeezes out more than one basic product, reducing the total number of firms.

This is not the end of the story, however: changes in exogenous fixed costs also affect quality investment and endogenous fixed costs (with no change in the cost of quality enhancement). By stimulating entry of premium goods, 
a reduction in $K$ induces each of the now-increased number of premium producers to invest less. This lowers their quality $v^{*}$, decreasing the degree of vertical differentiation between premium and basic products. It also lowers the demand facing an individual premium producer $x^{R}$, though the total market share of premium products $\left(1-S^{B}\right)$ still rises. The opposite changes occur following a reduction in $F$ : by encouraging entry of basic products, this inhibits entry of premium goods but increases investment in each one, raising their quality and widening the degree of vertical differentiation.

The findings for fixed cost reductions for basic products suggest that long tail and superstar phenomena can coexist. Lower $F$ raises the total number of products offered in the market, the proportion of these that are basic products, and the total market share of basic products - these are long tail outcomes. At the same time, the quality of premium products rises, as do their individual market shares - both superstar effects. Thus, a possible explanation for the observation of both long tail and superstar phenomena, as reported by Elberse and Oberholzer-Gee (2007) and Page and Garland (2009), is a reduction in the fixed costs of supplying basic products.

\subsubsection{Cost of quality enhancement}

As in Sutton (1991), endogenous fixed costs play an important role in determining market structure. A reduction in the cost of enhancing quality stimulates investment in premium products, increasing vertical differentiation. Endogenous fixed costs go up, reducing entry; hence the market provides less variety. The improved quality of premium products squeezes basic products, whose quality cannot be raised: these account for a smaller proportion of the now-reduced total number of products. With a higher proportion of premium products and greater investment in each, the total market share of premium products increases.

Thus, a lower cost of quality generates a superstar effect, in that there is more investment in the leading products and these provide higher qual- 
ity. Cheaper quality enhancement might explain the pattern observed in the movie industry in the latter half of the 20th century, when studios spent increasing amounts on production and blockbusters dominated the movie scene.

\subsubsection{Market size}

Changes in market size have a more limited range of effects. These have no impact on the equilibrium number of firms (as found in section 2.4), and do not change firms' quality and pricing strategies (contrary to the findings in section 2.4). Individual demands are unchanged, but since the size of the market is altered this entails that each product constitutes a smaller (for a growing market) or larger (for a shrinking market) share of the market. With endogenous product mix, the balance between premium and basic products is affected: market expansion increases the proportion and total market share of premium products, while a shrinking market similarly promotes basic products.

A declining market generates both long tail and superstar effects. Although there is no increase in the total number of products, a higher proportion of these are basic types (which individually achieve lower sales than premium products), and together these take a larger share of the marketlong tail outcomes. This might explain the changing distribution of music sales: while total sales have declined dramatically, the overall share accounted for by niche products has (by some accounts) markedly increased. Meanwhile in a shrinking market the sales levels of individual products hold up, implying that individual market shares rise. This could explain the finding of Elberse and Oberholzer-Gee (2007) for US home video sales - a market experiencing declining sales - that the top ten percent of sales is accounted for by a smaller number of top sellers - a superstar effect. 


\section{Concluding comments}

This paper has developed a modeling framework capturing the key features of creative media industries and used this to examine the impact of technological changes linked to digitization on market structure, product variety, quality investment, and product mix. It finds that a superstar effect in the sense of greater investment in the quality of top-selling products results from either lower quality costs for premium products or lower fixed costs for basic ones. A lower basic fixed cost also raises individual sales for these products (bigger hits) - another superstar effect. A decline in market size is found not to reduce sales levels for individual products, implying that the share of the now-smaller market held by given number of top sellers (say, the top ten) becomes larger, as seen in some of the empirical literature - also a superstar effect.

Long tail outcomes, in the sense of an increase in the number of basic products that are sold, are found to result from either a reduction in basic fixed costs or a smaller market. The former change increases the total number

of products, increasing variety, while the latter leaves the total number of products unchanged but increases the proportion of basic goods. In both cases the long tail is not merely an empty one: the total market share of basic products also rises.

The findings in this paper demonstrate that the coexistence of superstar and long tail phenomena - as found in a number of empirical studies - is possible, indeed likely. Either a reduction in basic fixed costs or a decline in market size can explain the observation of both types of outcome. A reduction in the cost of enhancing quality on the other hand is one-sided, generating superstar but not long tail effects.

The multitude and complexity of technological developments in creative media industries make predictions for the future highly uncertain. As these analyses show, future trends will be sensitive to the precise nature of changes brought about by new technologies. However, if the incidence of cost changes 
resulting from a particular technology can be assessed, the modeling in this paper can then provide guidance as to its likely impact on market structure and the distribution of product sales.

\section{Appendix: Proof of Proposition 2}

(i) Number of firms, $N^{*}=4 t \gamma \frac{(K-F)}{K}+1$.

$$
\begin{aligned}
\frac{d N^{*}}{d F} & =-\frac{4 \gamma t}{K}<0 . \\
\frac{d N^{*}}{d K} & =4 \gamma t \frac{F}{K^{2}}>0 . \\
\frac{d N^{*}}{d \gamma} & =4 t \frac{(K-F)}{K}>0 . \\
\frac{d N^{*}}{d M} & =0 .
\end{aligned}
$$

(ii) Proportion of premium products, $\lambda^{*}=\frac{F}{(K-F)}\left(\frac{M K}{(4 t \gamma(K-F)+K)} \sqrt{\frac{K}{2 \gamma(K-F) F}}-1\right)$.

$\frac{d \lambda^{*}}{d F}=\frac{K}{(K-F)^{2}} \frac{M G}{(4 t \gamma(K-F)+K)}\left(\frac{4 t \gamma\left(K^{2}-F^{2}\right)+K^{2}}{(4 t \gamma(K-F)+K)}+\frac{(2 F-K)}{2}\right)-\frac{K}{(K-F)^{2}}$ where $G=$ $\sqrt{\frac{K}{2 \gamma F(K-F)}}$. From (12) this has a lower bound where $\frac{M G}{(4 t \gamma(K-F)+K)}=\frac{1}{K}$. Thus $\frac{d \lambda^{*}}{d F} \geq \frac{4 \gamma t(4 F-K)(K-F)+K(2 F-K)}{2(K-F)^{2}(4 t \gamma(K-F)+K)}>0$ (given $\left.K<2 F\right)$.

$\frac{d \lambda^{*}}{d K}=-\frac{F}{(K-F)^{2}} \frac{M G}{(4 t \gamma(K-F)+K)}\left(\frac{4 t \gamma\left(K^{2}-F^{2}\right)+K^{2}}{4 t \gamma(K-F)+K}+\frac{F}{2}\right)+\frac{F}{(K-F)^{2}}$ where $G=\sqrt{\frac{K}{2 \gamma F(K-F)}}$.

From (12) this has an upper bound where $\frac{M G}{(4 t \gamma(K-F)+K)}=\frac{1}{K}$. Thus $\frac{d \lambda^{*}}{d K} \leq$ $-\frac{F^{2}(12 t \gamma(K-F)+K)}{2 K(K-F)^{2}(4 t \gamma(K-F)+K)}<0$.

$\frac{d \lambda^{*}}{d \gamma}=-\frac{M K}{(4 t \gamma(K-F)+K)} \sqrt{\frac{K}{2 \gamma F(K-F)}}\left(\frac{4 t F}{(4 t \gamma(K-F)+K)}+\frac{F}{2 \gamma(K-F)}\right)<0$.

$\frac{d \lambda^{*}}{d M}=\frac{F}{(K-F)} \frac{K}{(4 t \gamma(K-F)+K)} \sqrt{\frac{K}{2 \gamma(K-F) F}}>0$.

(iii) Quality of premium product, $v^{*}=\sqrt{\frac{2 K(K-F)}{\gamma F}}$.

$$
\begin{aligned}
& \frac{d v^{*}}{d F}=-\frac{K^{2}}{2 \gamma F^{2}} \sqrt{\frac{2 \gamma F}{K(K-F)}}<0 . \\
& \frac{d v^{*}}{d K}=\frac{(2 K-F)}{2 \gamma F} \sqrt{\frac{2 \gamma F}{K(K-F)}}>0 . \\
& \frac{d v^{*}}{d \gamma}=-\frac{K(K-F)}{2 \gamma^{2} F} \sqrt{\frac{2 \gamma F}{K(K-F)}}<0 . \\
& \frac{d v^{*}}{d M}=0 .
\end{aligned}
$$


(iv) Price of basic product, $p^{B *}=F \sqrt{\frac{K}{2 \gamma F(K-F)}}$.

$\begin{aligned} \frac{d p^{B *}}{d F} & =\frac{K^{2}}{4 \gamma(K-F)^{2}} \sqrt{\frac{2 \gamma(K-F)}{F K}}>0 . \\ \frac{d p^{B *}}{d K} & =-\frac{F^{2}}{4 \gamma(K-F)^{2}} \sqrt{\frac{2 \gamma(K-F)}{F K}}<0 . \\ \frac{d p^{B *}}{d \gamma} & =-\frac{K F}{4 \gamma^{2}(K-F)} \sqrt{\frac{2 \gamma(K-F)}{F K}}<0 . \\ \frac{d p^{B *}}{d M} & =0 .\end{aligned}$

(v) Price of premium product, $p^{R *}=K \sqrt{\frac{K}{2 \gamma F(K-F)}}$.

$\frac{d p^{R *}}{d F}=(2 F-K) \frac{K^{2}}{4 \gamma F^{2}(K-F)^{2}} \sqrt{\frac{2 F \gamma(K-F)}{K}}>0$ (given $\left.K<2 F\right)$.

$\frac{d p^{R *}}{d K}=(2 K-3 F) \frac{K^{2}}{4 \gamma F(K-F)^{2}} \sqrt{\frac{2 \gamma F(K-F)}{K}}<(>) 0$ for $K<(>) \frac{3}{2} F$.

$\frac{d p^{R *}}{d \gamma}=-\frac{K^{2}}{4 \gamma^{2} F(K-F)} \sqrt{\frac{2 \gamma F(K-F)}{K}}<0$.

$\frac{d p^{R *}}{d M}=0$.

(vi) Demand for a basic product, $x^{B}=\sqrt{2 \gamma \frac{F}{K}(K-F)}$.

$\frac{d x^{B}}{d F}=(K-2 F) \sqrt{\frac{\gamma}{2 K F(K-F)}}<0$.

$\frac{d x^{B}}{d K}=\frac{F}{K} \sqrt{\frac{F \gamma}{2 K(K-F)}}>0$.

$\frac{d x^{B}}{d \gamma}=\sqrt{\frac{F(K-F)}{2 \gamma K}}>0$.

$\frac{d x^{B}}{d M}=0$.

(vii) Demand for a premium product, $x^{R}=\sqrt{2 \gamma \frac{K}{F}(K-F)}$.

$\frac{d x^{R}}{d F}=-\frac{K}{F} \sqrt{\frac{\gamma K}{2 F(K-F)}}<0$.

$\frac{d x^{R}}{d K}=(2 K-F) \sqrt{\frac{\gamma}{2 F K(K-F)}}>0$.

$\frac{d x^{R}}{d \gamma}=\sqrt{\frac{K(K-F)}{2 F \gamma}}>0$.

$\frac{d x^{R}}{d M}=0$.

(viii) Total share of basic products, $S^{B}=\frac{4 t \gamma(K-F)+K}{(K-F)} \frac{1}{\sqrt{\frac{K}{2 \gamma F(K-F)}} M}-\frac{F}{(K-F)}$.

$\frac{d S^{B}}{d F}=\frac{1}{2 F(K-F) G M}\left(\frac{K^{2}}{(K-F)}-4 \gamma t(2 F-K)\right)-\frac{K}{(K-F)^{2}}$ where $G=\sqrt{\frac{K}{2 \gamma F(K-F)}}$. If $\left(\frac{K^{2}}{(K-F)}-4 \gamma t(2 F-K)\right)<0$ then $\frac{d S^{B}}{d F}<0$. If $\left(\frac{K^{2}}{(K-F)}-4 \gamma t(2 F-K)\right)>$ 
0, then from (12) the expression has an upper bound where $\frac{1}{G M}=\frac{K}{(4 \gamma t(K-F)+K)}$ : $\frac{d S^{B}}{d F} \leq-\frac{K(K(2 F-K)+4 \gamma t(4 F-K)(K-F))}{2 F(K-F)^{2}(4 \gamma t(K-F)+K)}<0$ (given $\left.K<2 F\right)$. Thus $\frac{d S^{B}}{d F}<0$. $\frac{d S^{B}}{d K}=\frac{F}{(K-F)^{2}}+\frac{F}{2 K(K-F)^{2}} \frac{1}{M G}(4 \gamma t(K-F)-K)$ where $G=\sqrt{\frac{K}{2 \gamma F(K-F)}}$. If $(4 \gamma t(K-F)-K)>0$ then $\frac{d S^{B}}{d K}>0$. If $(4 \gamma t(K-F)-K)<0$, then from (12) the expression has a lower bound where $\frac{1}{G M}=\frac{K}{(4 \gamma t(K-F)+K)}$ : $\frac{d S^{B}}{d K} \geq \frac{F(K+12 t \gamma(K-F))}{2(K-F)^{2}(K+4 t \gamma(K-F))}>0$. Thus $\frac{d S^{B}}{d K}>0$.

$\frac{d S^{B}}{d \gamma}=\frac{F}{K M}\left(\sqrt{\frac{K}{2 F K \gamma-2 F^{2} \gamma}}\right)(K+12 t \gamma(K-F))>0$. $\frac{d S^{B}}{d M}=-\frac{1}{(K-F) M^{2}} \frac{1}{\sqrt{\frac{K}{2 F K \gamma-2 F^{2} \gamma}}}(K+4 t \gamma(K-F))<0$.

\section{Acknowledgements}

The author would like to thank Simon Anderson, Mark Armstrong, Peter Davis, Pierre Régibeau, Paul Seabright, Andrew Sweeting, and seminar participants at Essex, Surrey, the Network of Industrial Economists Annual Meeting, the LECG Paris workshop on digital media, and the 6th International Workshop on Media Economics for helpful comments and discussion. The author also thanks the editors and two anonymous referees for their valuable comments and suggestions. Any errors remain the responsibility of the author. Financial support from the LECG/Microsoft grant program is gratefully acknowledged.

\section{References}

[1] Anderson, C., 2004. The long tail. Wired Mag. 12, 170-177.

[2] Anderson, C., 2006. The Long Tail. Random House Business Books, London.

[3] Bhaskar, V., To, T., 2004. Is perfect price discrimination really efficient? An analysis of free entry. RAND J. Econ. 35, 762-776. 
[4] Brito, D., 2003. Preemptive mergers under spatial competition. Int. J. Ind. Organ. 21, 1601-1622.

[5] Brynjolfsson, E., Hu, Y., Simester, D., 2011. Goodbye Pareto principle, hello long tail: the effect of search costs on the concentration of product sales. Manag. Sci. 57, 1373-1386.

[6] Elberse, A., Oberholzer-Gee, F., 2007. Superstars and underdogs: an examination of the long-tail phenomenon in video sales. Mark. Sci. Inst. 4, 49-72.

[7] Fleder, D., Hosanagar, K., 2009. Blockbuster culture's next rise or fall: the impact of recommender systems on sales diversity. Manag. Sci. 55, 697-712.

[8] Hotelling, H., 1929. Stability in competition. Econ. J. 39, 41-57.

[9] Page, W., Garland, E., 2009. The long tail of P2P. Econ. Insight 14. PRS for Music.

[10] Rosen, S., 1981. The economics of superstars. Am. Econ. Rev. 71, 845858.

[11] Salop, S.C., 1979. Monopolistic competition with outside goods. Bell J. Econ. 10, 141-156.

[12] Sutton, J., 1991. Sunk Costs and Market Structure. MIT Press, Cambridge MA.

[13] Tucker, C., Zhang, J., 2011. How does popularity information affect choices? A field experiment. Manag. Sci. 57, 828-842.

[14] Von Ungern-Sternberg, T., 1991. Monopolistic competition in the pyramid. J. Ind. Econ. 39, 355-368. 